\title{
LA GANADERÍA OVINA Y CAPRINA EN LA PROVINCIA DE GRANADA. REPERCUSIONES DE LA NUEVA PAC EN SU DISTRIBUCIÓN
}

\author{
Juan GÁMEZ NAVARRO*
}

\section{INTRODUCCIÓN}

Pretendemos realizar en este artículo una puesta al día de la situación por la que atraviesa la ganadería menor, ovina y caprina, en la provincia de Granada. Comenzamos por conocer la importancia que tuvo a mediados de siglo $\mathrm{XX}$, nos detenemos en analizar de forma pormenorizada las causas de la crisis ganadera que padeció el país, diferenciando la situación en cierto modo privilegiada que vivió la provincia de Granada, o mejor varias de su comarcas con más vocación ganadera, a diferencia de lo que ocurrió en Andalucía y España, donde la cabaña disminuyó de manera importante. Esa evolución diferenciada nos conduce a la situación actual caracterizada por un incremento de la cabaña en los últimos años; incremento superior al que se da en Andalucía y España, impulsado por las peculiares características de esta provincia y por las primas establecidas por la Unión Europea.

Este es el segundo gran objetivo del presente trabajo, cuantificar su importancia, tras analizar su evolución y determinar su posible influencia en la situación actual. A modo de ejemplo incluimos el valor de éstas primas, que en el año último han ascendido, en zonas desfavorecidas, a 5.360 pts. por oveja y a 4.162 por cabra.

Analizamos los aspectos fundamentales de la política de ayudas establecidas en la PAC, caracterizada por la compensación a la pérdida de valor de los productos cámicos, y consistente en una prima única para todos los productores de corderos pesados, y diferenciando según se trate de municipios situados en zonas desfavorecidas o de montaña, de aquellos situados en zonas «normales», que reciben menos ayudas. En este sentido es importante resaltar que el 93,34\% de la superficie de la provincia de Granada está considerada como zona desfavorecida, y, por tanto, recibe una prima adicional para compensar esta situación diferencial negativa.

* Doctor en Geografía. Universidad de Granada. 
A continuación entramos en el estudio de la estructura ganadera actual, estu diada en base a los Listados de cobro de las mencionadas primas en el año 199: de cuyo análisis podemos hacernos una idea bastante exacta de la situación y re parto de la cabaña: su volumen y distribución, de los problemas derivados de es estructura; así como de los tipos de propiedad predominantes.

Finalizamos con un análisis espacial, a nivel comarcal y municipal, que no acerca a la situación real de la cabaña, y nos permite hablar de áreas y municipio con cierta especialización ganadera, que en algunos casos proviene, al menos, desd mediados del s. XVIII.

\section{EVOLUCIÓN DE LA GANADERÍA MENOR EN LA PROVINCIA DE GRANADA}

Nos ceñimos a un análisis referido exclusivamente a la segunda mitad del s. XX

Los datos utilizados proceden de los censos ganaderos elaborados por el $\mathrm{Mi}$ nisterio de Agricultura hasta 1984, y a partir de esta fecha, de Ios datos publicado por el I.E.A. en el Anuario Estadístico de Andalucía. De ellas se desprenden do tendencias contrapuestas:

A) Una primera indica que la cabaña disminuyó entre 1960 y 1968, tal y comı ocurrió a nivel nacional donde decayó un 16,7\% entre 1967 y 1977, debid، a la dificultad para encontrar pastores como consecuencia del despoblamientı que padeció el campo, a la fuerte competencia de otros productos cámico y al deterioro de los precio de la lana; esta regresión fue acompañada de uı cambio en la orientación productiva ${ }^{1}$. Pero la crisis del sector ovino fue meno intensa en la provincia de Granada, como ha puesto de manifiesto A.L. Garcíi Ruiz, debido a que en las tres comarcas situadas al NE de la provincia, li ganadería ovina, es más productiva que la agricultura².

B) Una segunda fase de incremento continuado desde 1968, adelantándose li recuperación una década con respecto a España, y llegando a multiplicarsı por 2,3 la cabaña, según se desprende del último dato disponible, comı consecuencia de las buenas expectativas que tiene el subsector desde la in tegración de España en la U.E., y, especialmente, por la política de prima. puesta en marcha en los ochenta.

1. BRIZ ESCRIBANO, J. (1979): Política de precios y estructuras en el mercado de carnes: Referenci especial a la primas a la producción de añojos. Agricultura y Sociedad, n. ${ }^{9} 11$, pp. 149-206 y en concret. eu las pp. 186-187.

2. GARCÍA RUIZ, A.L. (1990): Situación de la ganadería en la provincia de Granada: composición distribución, sistemas de explotación y comercialización de productos. Universidad de Granada, pp. 34 


\section{CUADRO I}

EVOLUCIÓN DE LA GANADERÍA MENOR. PROVINCIA DE GRANADA, 1950-92

\begin{tabular}{|c|c|c|c|c|c|c|c|}
\hline \multirow{2}{*}{$\frac{\text { Año }}{1950^{*} \ldots \ldots \ldots \ldots}$} & \multicolumn{2}{|c|}{ Ovino (A) } & \multicolumn{2}{|c|}{ Caprino (B) } & \multirow{2}{*}{$\frac{\mathrm{A} / \mathrm{B}}{1,31}$} & \multirow{2}{*}{$\frac{\text { Total }}{378.931}$} & \multirow{2}{*}{$\frac{1950=100}{100,00}$} \\
\hline & 214.835 & 100,00 & 164.096 & 100,00 & & & \\
\hline $1955^{*} \ldots \ldots \ldots \ldots$ & 222.573 & 103,60 & 152.615 & 93,00 & 1,46 & 375.188 & 99,01 \\
\hline $1960 \ldots \ldots \ldots \ldots . . . . . .$. & 316.684 & 147,41 & 214.096 & 130,47 & 1,48 & 530.780 & 140,07 \\
\hline 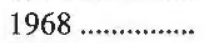 & 307.875 & 143,31 & 110.008 & 67,04 & 2,80 & 417.883 & 110,28 \\
\hline $1974 \ldots \ldots . . . . . . . . . . .$. & 364.712 & 169,76 & 119.792 & 73,00 & 3,04 & 484.504 & 127,86 \\
\hline $1984^{1} \ldots \ldots \ldots \ldots . . . . .$. & 382.098 & 177,86 & 129.254 & 78,77 & 2,96 & 511.352 & 134,95 \\
\hline $1986^{1} \ldots \ldots \ldots \ldots$ & 369.450 & 171,97 & 146.456 & 89,25 & 2,52 & 515.906 & 136,15 \\
\hline $1988^{1} \ldots \ldots \ldots \ldots . . . .$. & 527.598 & 245,58 & 262.036 & 159,68 & 2,01 & 789.634 & 208,38 \\
\hline $1990^{1} \ldots \ldots \ldots \ldots . . . .$. & 542.397 & 252,47 & 259.926 & 158,40 & 2,09 & 802.323 & 211,73 \\
\hline $1992^{1} \ldots \ldots \ldots \ldots$ & 681.601 & 317,27 & 188.423 & 114,82 & 3,62 & 870.024 & 229,60 \\
\hline
\end{tabular}

Fuentes: M.ำ de Agricultura. Censos Ganaderos.

* Estos censos no incluyeron los animales de menos de un año.

1. Anuario Estadístico de Andalucía.

Sin embargo, este crecimiento no afecta por igual a las dos especies, pues mientras que el ovino ha pasado de 214.835 cabezas en 1950 a 681.601 en 1992; es decir, se ha incrementado un 217,3\%; en cambio, el caprino ha pasado de 164.096 cabezas a 188.423; es decir, sólo ha crecido un 14,8\%, si bien es cierto que en 1988 alcanzó su máximo numérico, y, desde ese año no ha dejado de reducirse.

En efecto, se corrobora una tendencia cualitativa, que es hacia el predominio del ganado ovino, en detrimento del caprino, ya que su proporción en 1950 era de 1,3 cabezas de ovino por 1 de caprino, mientras que al final del período considerado esta proporción se ha triplicado, lo que significa una pérdida de importancia de éste en favor de aquél, debida al cambio de orientación de la cabaña, que desde los años ochenta se orienta más hacia la producción de came ${ }^{3}$ que a la de leche, a la que se dedicaba casi en su totalidad la cabaña caprina, como orientación principal, por el predomino de la raza murciano-granadina, una de las más conocidas por su especialización en la producción de leche ${ }^{4}$ y dadas las necesidades de producción de ésta para su uso inmediato a nivel doméstico, hoy salvada esta dependencia de la producción propia para la alimentación familiar por la comercialización fácil y rápida de la leche de vaca, lo que unido a la mayor cuantía que tienen las primas al ovino, han determinado este incremento de la cabaña del ovino, que triplica a la del ganado caprino.

3. BRIZ ESCRIBANO, J. op. cit. pp. 187.

4. GARCÍA DORY, M.A. (1980): La utilización de las razas autóctonas en los ecosistemas regionales como factor de ahorro energético en la ganadería española. Agricultura y Sociedad, nº 15, pp. 116-161. 
Esta tendencia al incremento de la cabaña ganadera, especialmente de la ovin tiene que ver, con la aprobación del Real Decreto 464/79, que pretendió foment la ganadería extensiva en zonas de montaña, y que «viene a corregir la tendenci regresiva de la ganadería en amplias zonas del país manifiestamente infrautilizad en contraste con el desarrollo de la ganadería industrial» ${ }^{5}$. No olvidemos que un de las características de esta especie radica precisamente en su capacidad de aprc vechamiento de los recursos marginales de nuestro secano, de las extensas zona de erial-matorral existentes en el sureste peninsular y su fuerte conexión con 1 empresa agrícola ${ }^{6}$. En los últimos años, las primas de la U.E. han contribuido tarr bién a su incremento, salvándose así la crisis que afectó al sector en los momentc centrales del siglo, cuando la agricultura era la niña mimada de la administraciór debido a la escasez de recursos alimenticios, hecho que influyó decisivamente e que el gobiemo emprendiera una política cerealista, y, en particular, favorecedor del cereal panificable, el trigo.

En el año 1950 el censo de ganado ovino de Granada suponía el 13,4\% del tot: andaluz; en cambio, veinticuatro años más tarde la relación se ha incrementadc pasando a representar el $22 \%$, lo que supone un crecimiento de casi diez punto: disminuyendo a partir de este año para situarse finalmente en el 20,2\% en 1992

El crecimiento de la cabaña tiene su punto culminante en 1992 en ambas un: dades, siendo en Granada más intenso, ya que ha duplicado el número de cabeza: mientras en Andalucía el incremento ha sido del $90 \%$ y en España del 43.

\section{CUADRO II-A \\ EVOLUCIÓN DEL GANADO OVINO EN GRANADA, ANDALUCÍA Y ESPAÑA, 1950-92}

\begin{tabular}{|c|c|c|c|c|c|c|c|}
\hline \multirow{2}{*}{$\frac{\text { Año }}{1950^{1} \ldots \ldots \ldots \ldots \ldots . . . . . .}$} & \multicolumn{2}{|c|}{ Granada (A) } & \multicolumn{2}{|c|}{ Andalucía (B) } & \multirow{2}{*}{$\frac{\mathrm{A} / \mathrm{B} * 100}{13,36}$} & \multicolumn{2}{|c|}{ España $(\mathrm{C})$} \\
\hline & 214.835 & 100,00 & 1.642 .448 & 100,00 & & 16.344 .000 & 100,00 \\
\hline 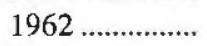 & 188.759 & 116,25 & 2.272 .546 & 138,36 & 14,49 & 22.622 .000 & 138,41 \\
\hline 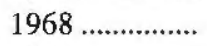 & 307.875 & 110,28 & 1.977 .315 & 120,39 & 18,15 & 16.726 .000 & 102,34 \\
\hline 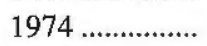 & 364.712 & 127,86 & 1.506 .539 & 91,73 & 21,92 & 15.598 .524 & 95,44 \\
\hline $1984 \ldots \ldots . . . . . . . . . .$. & 382.098 & 134,95 & 1.923 .526 & 117,11 & 18,48 & 17.052 .675 & 104,34 \\
\hline $1990 \ldots \ldots . . . . . . . .$. & 542.397 & 211,73 & 3.186 .345 & 194,00 & 17,02 & 24.037 .000 & 147,07 \\
\hline $1992^{2} \ldots \ldots \ldots \ldots$ & 681.601 & 229,60 & 3.144 .061 & 191,43 & 20,17 & 23.371 .000 & 142,99 \\
\hline
\end{tabular}

Fuentes: Censos Ganaderos y Anuarios Estadístico de Andalucía y de España.

1. El censo de 1950 excluyó las crías. Para Andalucía los datos corresponden al censo de 1948.

2. Para España los datos corresponden al año 1991.

5. RODRÍGUEZ ZÚNIIGA, M. et al. (1980): El desarrollo ganadero español: un modelo dependiente desequilibrado. Agricultura y Sociedad, n.ํ1, pp. 165-193.

6. BRIZ ESCRIBANO, J. op. cit. pp. 189. 
La tendencia en Granada, salvo en los años centrales de los sesenta es al incremento, duplicándose el censo inicial en 1990, mientras que en Andalucía la tendencia fue contraria entre los años 1962 y 1974, años en que disminuye el censo hasta el punto de quedar por debajo del inicial; pero a partir de la década de los ochenta la cabaña tiende de nuevo al incremento. Esa crisis ganadera se aprecia de igual forma en España. El incremento más fuerte de la cabaña ovina se ha producido en la provincia de Granada, donde se ha multiplicado por 2,3 veces; en cambio, en Andalucía y en España este incremento ha sido de menor intensidad. Por contra, en el ganado caprino el crecimiento mayor se ha producido en Andalucía, seguida de Granada, mientras en España disminuye, como se aprecia en el cuadro II-B.

\section{CUADRO II-B \\ EVOLUCIÓN DEL GANADO CAPRINO EN GRANADA, ANDALUCÍA Y ESPAÑA, 1950-92}

\begin{tabular}{|c|c|c|c|c|c|c|c|}
\hline \multirow{2}{*}{ Año } & \multicolumn{2}{|c|}{ Granada (A) } & \multicolumn{2}{|c|}{ Andalucía (B) } & \multirow{2}{*}{$\mathrm{A} / \mathrm{B} * 100$} & \multicolumn{2}{|c|}{ España (C) } \\
\hline & Total & $\%$ & Total & $\%$ & & Total & $\%$ \\
\hline $1950^{1}, \ldots \ldots \ldots \ldots . . . . .$. & 164.096 & 100,00 & 641.484 & 100,00 & 25,58 & 4.135 .000 & 100,00 \\
\hline $1962 \ldots . . . . . . . . . .$. & 114.313 & 69,66 & 766.855 & 119,54 & 18,01 & 3.300 .000 & 79,81 \\
\hline $1968 \ldots \ldots \ldots \ldots . . . . . . .$. & 110.008 & 67,04 & 747.447 & 116,52 & 17,15 & 2.504 .000 & 60,56 \\
\hline 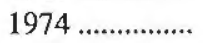 & 119.782 & 73,00 & 703.681 & 109,70 & 17,02 & 2.230 .000 & 53,93 \\
\hline 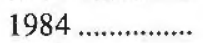 & 129.524 & 78,93 & 845.022 & 131,73 & 15,33 & 2.533 .000 & 61,26 \\
\hline 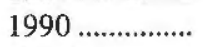 & 259.926 & 158,40 & 1.528 .115 & 238,22 & 17,01 & 3.663 .000 & 88,59 \\
\hline $1992^{2} \ldots \ldots \ldots \ldots . . . . . .$. & 188.423 & 114,82 & 1.169 .154 & 182,26 & 16,12 & 2.972 .000 & 71,87 \\
\hline
\end{tabular}

Fuente: Censos Ganaderos y Anuarios Estadístico de Andalucía y de España.

1. El censo de 1950 excluyó las crías. Para Andalucía los datos corresponden al censo de 1948.

2. Para España los datos corresponden al año 1991.

La evolución del ganado caprino es claramente distinta a la del ovino, especialmente en la provincia de Granada, en donde constatamos una profunda crisis, que alcanza su punto mínimo en 1968; desde esa fecha hasta la década de los noventa en que vuelve a superar las cifras iniciales de 1950 no ha dejado de crecer, pero de nuevo en 1992 vuelve a disminuir. En Andalucía la cabaña ha tenido una evolución diferenciada, ya que siempre ha sido una cabaña superior a la de 1950, aunque en los sesenta se notó algo la crisis, pero en 1990 multiplica la cabaña por 2,4 veces la inicial, si bien los datos finales vuelven de nuevo a marcar una intensa disminución. La situación a nivel nacional es de claro retroceso de la cabaña, llegando en los años cincuenta a reducirse a la mitad, en la actualidad el número de cabezas representa el $71,9 \%$ del inicial. 
La crisis de la ganadería extensiva andaluza ${ }^{7}$ y española ${ }^{8}$, no tuvo una clar: repercusión en la provincia de Granada ${ }^{9}$, donde posiblemente no se daban las mis mas circunstancias que en otras áreas andaluzas, al ser esta provincia de montaña fundamentalmente, y, por tanto, con abundantes terrenos sólo aptos para el uso : diente de su producción herbácea por los rebaños de cabras y ovejas, a pesar di que la superficie provincial susceptible de ser utilizada para pastos directos signi fica un porcentaje inferior (45\%) al disponible a nivel nacional (53\%) (ver cuadr V). Sin embargo, hay otro factor que puede resultar decisivo, como es el ya men cionado relativo a que casi el $87 \%$ de la superficie provincial está considerada zon: desfavorecida de montaña, lo que motiva que las primas recibidas sean superiores Otros factores en opinión de A.L. García Ruiz son el fenómeno que se produce es las comarcas de Baza, Huéscar y Guadix, comarcas en las que la explotación de li ganadería ovina aumenta, mientras la agricultura disminuye, porque el carácte extremo y seco del clima y la poca calidad del suelo hace a la agricultura meno. rentable que la ganadería. A ello se une el desarrollo del turismo, que hizo aumen tar la demanda de carne de cordero en las próximas zonas del levante español ${ }^{10}$

La recuperación de la cabaña española de ovino en los últimos años tiene qu ver mucho con la incorporación de España a la U.E. donde debido a que hay ur déficit de carne, se han establecido subvenciones que se han pagado desde el in greso en la comunidad a los ganaderos en compensación de los bajos precios es pañoles. El incremento del ovino es más intenso que el del caprino, cuyo com petidor más importante es la repoblación forestal de zonas de pastos pobres d montaña, que es el ámbito donde esta cabaña tenía más importancia ${ }^{11}$.

Aunque en ella se incluye toda la ganadería, y no sólo la menor, que es el objete de análisis de este artículo, creemos que puede ser interesante su inclusión a efec tos de comprobar cómo ha evolucionado su importancia económica en el context general del valor de la producción agrícola; es decir, si ha incrementado o dismi nuido su aportación al valor final de la producción agraria.

La producción final ganadera ha tenido un fuerte retroceso en la provincia d Granada, pasando del $26 \%$ en 1964 , año en que alcanza su valor porcentual má: elevado, al 12,63 en 1991. Ha sido muy inferior a la que aporta a nivel nacional ! algo inferior a la andaluza, como se desprende del cuadro III. Similar disminuciór se ha producido en Andalucía, donde ha pasado de suponer el $26 \%$ a poco más de 13\%. En España no ha cesado de incrementarse esta participación hasta 1975

7. GODOY LÓPEZ, L. (1979): La ganadería andaluza. Instituto de Desarrollo Regional. Universidar de Granada. Granada, pp. 55-58.

8. GARCÍA DORY, M.A. y MARTÍNEZ VICENTE, S. (1988): La ganadería en España. Alianz: Editorial, Madrid, pp. 58.

9. GARCIA RUIZ. A.L. op. cit pp. 33-35.

10. GARCÍA RUIZ, A.L. op. cit. pp. 35.

11. INSTITUTO GALLACH (1994): Geografía de España. Volumen V; Ed. Océano, Madrid, pp, 911 
manifestando una tendencia opuesta a la que se ha producido en Andalucía y en la provincia de Granada. En todo caso en 1992 aún el valor de la aportación de la ganadería a la producción final agraria en España casi triplica al que tiene en la provincia de Granada.

CUADRO III

EVOLUCIÓN DE LA PRODUCCIÓN GANADERA

(\% SOBRE LA PRODUCCIÓN FINAL AGRARIA)

\begin{tabular}{|c|c|c|c|}
\hline Año & Granada & Andalucía & España \\
\hline 1955 & 21,29 & 25,85 & 31,54 \\
\hline 1960 & 22,08 & 21,00 & 29,79 \\
\hline 1964 & 26,12 & 27,80 & 33,67 \\
\hline 1971 & 24,88 & 23,73 & 38,04 \\
\hline 1975 & 20,74 & 21,12 & 39,34 \\
\hline 1981 & - & - & 42,40 \\
\hline 1983 & 13,60 & 16,80 & 42,90 \\
\hline 1985 & 20,70 & - & 39,40 \\
\hline 1991 & 12,63 & 13,48 & 35,00 \\
\hline
\end{tabular}

Fuente: Banco de Bilbao y Anuario Estadístico de Andalucía.

\section{SUPERFICIE NO LABRADA Y APROVECHAMIENTOS}

La superficie no labrada, pero productiva, disponible para la alimentación a diente del ganado permite establecer las siguientes conclusiones:

a) Mientras que en Granada la superficie predominante es la labrada (48,1\%), en España el porcentaje es inferior en casi 9 puntos (39,7\%). En Andalucía la distribución es muy similar a la de Granada (cuadro IV).

b) En España predomina claramente la superficie no labrada, con el $53 \%$ del total, es decir, tiene una mayor disponibilidad de superficie para ganado ya que se trata de tierras de posible uso ganadero, con pastos o sin él. De hecho si utilizamos los datos del Censo Agrario (cuadro V) la proporción de tierras dedicadas a pastos permanentes es superior en 7 puntos en España a la que hay en Granada.

La producción en este período ha permanecido muy estancada en Andalucía en lo que se refiere a la producción de carne, mientras en Granada ha aumentado casi un 7\%. El incremento mayor de la producción corresponde a la producción lechera, especialmente en Andalucía donde se ha incrementado un $60 \%$; en cambio, en Granada sólo lo hizo en un $15 \%$. La producción de lana apenas ha experimentado un 4\% de incremento en Granada, por un I6 en Andalucía (cuadro VI). 


\section{CUADRO IV \\ PROPORCIÓN DE LA SUPERFICIE NO LABRADA RESPECTO DE LA TOTAL EN 1991 (MILES DE HA.)}

\begin{tabular}{|c|c|c|c|c|c|c|c|}
\hline & \multicolumn{2}{|c|}{ Superficie labrada } & \multicolumn{2}{|c|}{ Superficie no labrada } & \multicolumn{2}{|c|}{ Improductiva } & \multirow{2}{*}{ Total } \\
\hline & Total & $\%$ & Total & $\%$ & Total & $\%$ & \\
\hline Granada ........... & 608,4 & 48,11 & 571,8 & 45,21 & 84,5 & 6,68 & 100,00 \\
\hline Andalucía ....... & $4.174,5$ & 47,65 & $4.059,6$ & 46,34 & 525,8 & 6,01 & 100,00 \\
\hline España ............ & $20.088,3$ & 39,70 & $26.804,9$ & 52,98 & $3.705,8$ & 7,32 & 100,00 \\
\hline
\end{tabular}

Fuente: I.N.E. Anuario Estadístico de España, 1994. Capítulo X, apartado 1.1.1.

\section{CUADRO V \\ PROPORCIÓN DE LA SUPERF1CIE DEDICADA A PASTOS RESPECTO DE LA TOTAL EN 1991 (MILES DE HA.)}

\begin{tabular}{|c|c|c|c|c|c|c|c|}
\hline & \multicolumn{2}{|c|}{ Tіепаs labradas } & \multicolumn{2}{|c|}{$\begin{array}{c}\text { Tierras para } \\
\text { pastos permanentes }\end{array}$} & \multicolumn{2}{|c|}{ Otras tierras } & \multirow{2}{*}{ SAU } \\
\hline & Total & $\%$ & Total & $\%$ & Total & $\%$ & \\
\hline Granada ........... & 510.796 & 44,78 & 147.231 & 12,91 & 482.530 & 42,31 & 658.027 \\
\hline Andalucía ....... & 3.388 .190 & 44,61 & 1.149 .002 & 15,13 & 3.058 .415 & 40,27 & 4.537 .192 \\
\hline España ............. & 16.247 .700 & 37,84 & 8.492 .796 & 19,78 & 18.198 .701 & 42,38 & 24.740 .496 \\
\hline
\end{tabular}

Fuente: I.N.E. Anuario Estadístico de España, 1994. Datos procedentes del Censo Agrario.

\section{CUADRO VI \\ PRODUCCIÓN DE CARNE, LECHE Y LANA DEL GANADO OVINO Y CAPRINO}

\begin{tabular}{lrrrrrr}
\hline & \multicolumn{2}{c}{ Came (Tm.) } & \multicolumn{2}{c}{ Leche (miles L.) } & \multicolumn{2}{c}{ Lana (Tm.) } \\
\cline { 2 - 7 } Año & Granada & Andalucía & Granada & Andalucía & Granada & Andalucía \\
\hline & 783,90 & $7.786,80$ & 30.078 & 129.768 & 540,7 & $2.654,2$ \\
$1983 \ldots \ldots . . . .$. & 836,90 & $7.806,40$ & 34.436 & 207.283 & 563,0 & $3.083,0$ \\
$1992 \ldots . . . . . . . . . .$. & 106,76 & 100,25 & 114,49 & 159,73 & 104,12 & 116,16 \\
\hline
\end{tabular}

Fuente: I.E.A. Anuario Estadístico de Andalucía. 
La producción de came de ovino ha decrecido, pasando de 532 a 499 Tm.; en cambio, la de caprino se ha incrementado, pasando de $252 \mathrm{Tm}$. a 338 . El número de corderos sacrificados en 1992 fue de 43.978, de ellos la inmensa mayoría (40.330) del tipo pascual, que tienen un peso medio por canal de $12 \mathrm{kgs.;}$ en cambio, las reses de caprino sacrificadas ascienden a 67.678 , la mayoría $(58.171)$ coresponden al tipo cabrito lechal, con un peso medio de $4,7 \mathrm{kgs}$. en la provincia de Granada.

En la producción de leche se invierte la situación, ya que la inmensa mayoría procede del ganado caprino, muy especializado en esta producción, especialmente en la provincia de Granada, donde la raza granadina tiene una fuerte presencia.

De la producción de lana del año 1992 en Granada se reparten casi al 50\% entre la Blanca Entrefina y la Blanca Basta $(280 \mathrm{Tm}$. de cada variedad, por sólo 3 del tipo Negra). En Granada no hay producción del tipo Blanca fina. Para Andalucía el tipo predominante es el Blanca Entrefina (2.161 Tm.). En 1983 la mayoría de la lana producida correspondía al tipo Blanca Basta (482 Tm.).

\section{REPERCUSIONES DE LA PAC: LAS PRIMAS AL OVINO Y CAPRINO}

Las primas al sector ganadero, y, en particular, a la ganadería menor (ovino y caprino) se establecieron a comienzos de los años ochenta ${ }^{12}$ y han evolucionado como se recoge en el cuadro VII. Éstas son establecidas por la Comisión europea cada año, en función de los precios que ha alcanzado el cordero en los mercados de la Unión durante los meses anteriores, y con la finalidad de compensar las rentas de los productores de carne de ovino y caprino, que tienen 10 o más cabezas ${ }^{13}$, esta pérdida se calcula por $100 \mathrm{kgs}$. de peso en canal y es igual a la diferencia entre el precio base y la media aritmética de los precios de mercado registrados en cada una de las regiones. El importe se obtiene aplicando a la pérdida de renta un coeficiente que representa, para cada región, la producción media anual de carne de cordero por oveja expresada por $100 \mathrm{kgs}$. de peso en canal.

La prima se paga la final de la campaña, sin embargo, si durante la campaña se observa que puede producirse una pérdida de renta importante, los Estados miembros afectados pueden conceder un anticipo a cuenta del $30 \%$ de la prima estimada, liquidándose al final de la campaña, que es cuando se conoce el importe definitivo de la misma.

La evolución de estas primas, recogida en el cuadro VII, pone de manifiesto que se han duplicado, pasando, en las zonas desfavorecidas, de 2.565 y 2.052 pts.

12. Las ayudas a los productores de carne de ovino se establecieron en los Reglamentos $1837 / 80$ y $872 /$ 84; y a los productores de carne de caprino en el Reglamento 3523/85.

13. MAPA (1986): Ayudas de la Comunidad Económica Europea al sector agrario. Madrid, pp. 37. 
al ovino y caprino respectivamente, a 5.360 y 4.162 en los años consignados en e cuadro, pero en realidad habría que descontar el efecto de la inflación, que no hemos tenido en cuenta, para conocer realmente el incremento real de la subvención. Er las zonas normales la ayuda es siempre menor, un $20 \%$ aproximadamente, pero si incremento ha supuesto que en estos ocho años estudiados se duplique. La evolución no ha sido lineal sino que se han producido importantes reducciones, desta. cando la de 1989 debido a la puesta en marcha del mecanismo estabilizador pre. visto para cuando se superase la cabaña total con derecho a prima, que es la de: censo de 1987.

\section{CUADRO VII \\ EVOLUCIÓN DE LAS PRIMAS PAGADAS AL GANADO OVINO Y CAPRINO, 1987-1995}

\begin{tabular}{|c|c|c|c|c|}
\hline \multirow{2}{*}{ Año } & \multicolumn{2}{|c|}{ Zona Desfavorecida } & \multicolumn{2}{|c|}{ Zona Normal } \\
\hline & Ovino & Caprino & Ovino & Caprino \\
\hline 1987 ......................... & 2.565 & 2.052 & - & - \\
\hline $1988 \ldots$ & 2.349 & 1.880 & - & - \\
\hline 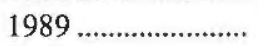 & 1.993 & 1.594 & 1.594 & 1.275 \\
\hline $1990 \ldots$ & 3.654 & 2.558 & 2.558 & 1.791 \\
\hline 1991 & 3.730 & 2.611 & 3.119 & 2.183 \\
\hline $1992 \ldots$ & 4.254 & 3.287 & 3.091 & 2.473 \\
\hline 1993 & 5.025 & 3.096 & 3.096 & 1.907 \\
\hline 1994 & 4.511 & 3.492 & 3.445 & 2.757 \\
\hline 1995 & 5.360 & 4.162 & 4.271 & 3.410 \\
\hline Incremento $\%$........ & 208,9 & 202,83 & 267,94 & 267,45 \\
\hline
\end{tabular}

Fuente: Delegación Provincial de Agricultura y Pesca. Desarrollo Ganadero.

Esta prima fue en el año 1995 de 4.271 pts. para el ovino y de 3.410 para el caprino; pero, además, se ha establecido una ayuda especial sólo para los productores de corderos pesados que están censados en las Zonas Desfavorecidas, por razón de sus especiales condiciones negativas para la producción, que en el año 1995 ha sido de $1.089 \mathrm{pts}$. para el ovino ${ }^{14}$ y de 752 para el caprino que sumadas a las

14. Prima para los productores de corderos pesados que se aplica a todas las hembras de la especie ovina o caprina que hayan sido cubiertas por primera vez; así como a toda hembra que haya parido por lo menos una vez, a excepción de las destinadas a desvieje, y que aún están presentes en la explotación. En general son las que tiene más de 1 año, excluyendo las de desecho. Los productores de corderos ligeros, que son aquellos que comercializan la leche o sus derivados lácteos, reciben el $80 \%$ del valor correspondiente a la prima recibida por los productores de corderos pesados. 
anteriores dan un total de 5.360 y 4.162 pts. respectivamente. En el año 1994 la prima suplementaria para las zonas desfavorecidas ascendió a 1.065,27 y 735,47 pts. respectivamente.

¿Qué zonas son desfavorecidas en la provincia de Granada? En la provincia de Granada (ver cuadro VIII y figura 1) prácticamente la totalidad de los municipios están considerados como pertenecientes a zonas desfavorecidas, bien por tratarse de áreas de montaña o por razón de tener un despobIamiento acentuado, como ocurre en varios municipios de las comarcas de Baza (Benamaurel, Cortes y Freila); de Guadix (Benalúa, Huélago y FoneIas); de Alhama (Chimeneas, Escúzar, Malá y Ventas de Huelma) y de Huéscar (Castillejar y Galera). Solamente hay una comarca, la de la Vega, en la que hay un total de 23 municipios ${ }^{15}$ que no tienen esta consideración. En su conjunto la provincia de Granada tiene el 93,34\% de su superficie comprendida en el grupo de áreas desfavorecidas, con las ventajas de índole agrícola y ganadero que tienen reconocidas por la U.E.

\section{CUADRO VIII}

COMARCAS DE GRANADA. ÁREAS DESFAVORECIDAS DE MONTAÑA Y POR DESPOBLAMIENTO (HA.)

\begin{tabular}{|c|c|c|c|c|c|}
\hline \multirow{2}{*}{ Comaca } & \multicolumn{3}{|c|}{ Zonas Desfavorecidas } & \multirow{2}{*}{ Resto } & \multirow{2}{*}{ Total } \\
\hline & Montaña & Despobl. & Total & & \\
\hline De la Vega ........... & 116.665 & 0 & 116.665 & 83.518 & 200.183 \\
\hline 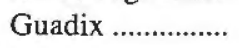 & 170.862 & 13.499 & 184.361 & 0 & 184.361 \\
\hline 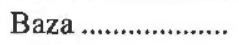 & 135.614 & 33.614 & 169.229 & 0 & 169.229 \\
\hline Huéscar ............... & 154.520 & 24.080 & 178.600 & 0 & 178.600 \\
\hline Iznalloz ................. & 86.984 & 33.349 & 120.333 & 0 & 120.333 \\
\hline Montefrío ............ & 65.926 & 0 & 65.926 & 0 & 65.926 \\
\hline Alhama ................ & 76.748 & 19.960 & 96.708 & 0 & 96.708 \\
\hline La Costa .................. & 77.278 & 0 & 77.278 & 0 & 77.278 \\
\hline Las Alpujarras... & 113.570 & 0 & 113.570 & 0 & 113.570 \\
\hline Valle Lecrín........ & 46.947 & 0 & 46.947 & 0 & 46.947 \\
\hline Total ........................... & 1.045 .114 & 124.503 & 1.169 .617 & 83.518 & 1.253 .135 \\
\hline
\end{tabular}

Fuente: I.N.E. y Pezzi, M. La comarcalización de Andalucía.

15. Estos municipios son: Albolote, Alhendín, Armilla, Atarfe, Cajar, Calicasas, Cijuela, Cúllar-Vega, Chauchina, Churriana de la Vega, Fuente Vaqueros, Las Gabias, Granada, Huétor-Tájar, Jun, Láchar, Maracena, Moraleda de Zafayona, Ogíjares, Otura, Peligros, Pinos-Puente, Salar, Santa Fe, Vegas del Genil y Villanueva de Mesía. 
FIGURA 1

PROVINCIA DE GRANADA. ZONAS DESFAVORECIDAS

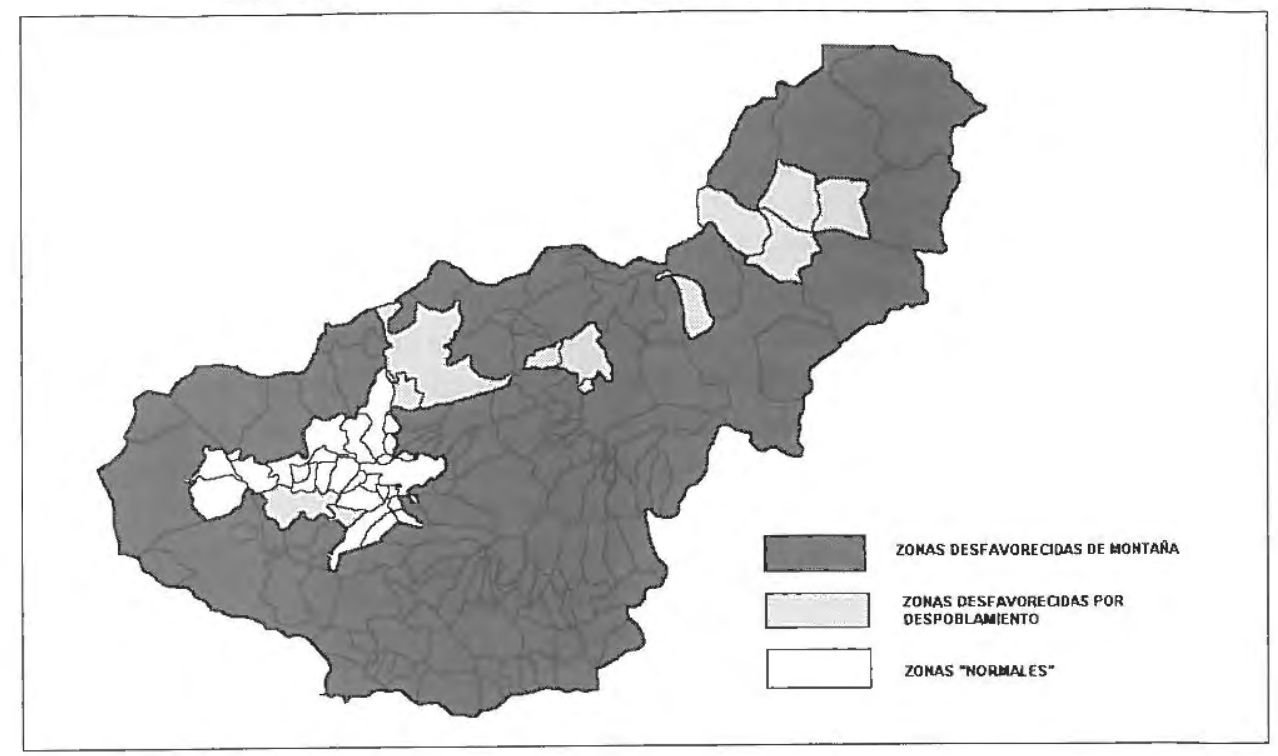

Solamente el 6,66\% de la provincia de Granada no está considerado como zona desfavorecida, municipios todos ellos situados en la Vega de Granada; el 83,41\% está considerado como zona de montaña y el $9,94 \%$ restante como zona desfavorecida por razón de su despoblamiento. Por comarcas, excepto la de la Vega, donde se sitúan los únicos términos municipales no incluidos en zonas desfavorecidas, destacan las de Montefrío, La Costa, Las Alpujarras y Valle de Lecrín que tienen todos sus municipios considerados como de montaña; en cambio, en las de Baza, Guadix, Huéscar, Iznalloz y Alhama hay algunos municipios considerados desfavorecidas por haber perdido mucha población, pero que a la postre supone los mismos derechos de cobro.

Cara al cobro de las primas concedidas a los productores de carne de ovino y caprino tienen el mismo tratamiento todas las zonas desfavorecidas, en consecuencia casi el $95 \%$ de la superficie provincial recibe estas primas de compensación a la pérdida de renta que padecen estos ganaderos.

La consecuencia más evidente de la PAC es el incremento de la renta de las explotaciones, especialmente de las dedicadas a la producción de corderos pesados, ya que la prima es un $20 \%$ superior. Paulino Navalpotro ${ }^{16}$ ha estimado un

16. NAVALPOTRO JiMÉNEZ, P. (1994): Explotaciones de ovino en los montes de Ciudad Real: viabilidad económica e impactos de la reforma de la PAC. Estudios Geográficos, n.² 214, pp. 109-133. 
incremento de la renta en las explotaciones de entre el 20 y el 35\%, siendo el incremento mayor en las explotaciones de corderos pesados, que son las más rentables gracias a la disminución de los costes de alimentación. Las ayudas compensan la pérdida de poder adquisitivo debido a la caída de los precios que han sufrido las productos agroganaderos, que asciende a 1,2 en los corderos, a 2,4 y 5,7 en el ovino mayor y en la lana ${ }^{17}$; como elemento positivo refiere la caída de los precios de los piensos y como negativo la subida de la mano de obra, que es 1,5 veces más cara que en 1972.

\section{LA ESTRUCTURA GANADERA ACTUAL}

Consideramos el estudio conjunto de la estructura ganadera (ovino y caprino) ya que los rebaños son una unidad de explotación donde se mezclan ambos tipos de ganado, aunque como también las hay de ovino y caprino exclusivamente las analizaremos también aparte. Los datos los hemos obtenido de los Listados de cobro de las primas al ovino y caprino.

Para el año 1993 la provincia recibió un total de 2.506,4 millones de pts. por este concepto lo que supone un incremento significativo de la renta ganadera, ya que como media cada ganadero recibe 742.425 pts., con una cabaña media de 166 cabezas, que en realidad sería mayor ya que las primas no las reciben más que a las hembras de más de 1 año que hayan sido cubiertas al menos una vez, cuando treinta años atrás la media era de 21,8 (Censo Agrario de 1962), que se había incrementado hasta 66 en 1982; es decir, constatamos un incremento sustancial del número medio de animales por rebaño, debido a la necesidad de incrementar el número de éstas para hacer frente a la subida del salario de los pastores, que en muchos casos han accedido a la propiedad del rebaño. No obstante, las diferencias de renta son sustanciales, como se desprende del cuadro IX, en el que estudiamos la estructura ganadera resultante de los listados de explotaciones por municipios correspondientes al año 1993.

Casi la mitad de las explotaciones, el 44,8\%, tiene menos de 101 cabezas, lo que nos hace pensar en la pervivencia de un sistema de explotación familiar muy ligado a las prácticas tradicionales, en las que se compaginan las labores agrícolas y las ganaderas, entendiéndose éstas como complemento de las primeras, hecho que viene corroborado porque el $22,4 \%$ tienen menos de 51 cabezas, explotaciones poco viables económicamente. Según García Ruiz en 1984 predominaba en nuestra provincia el tipo de explotación familiar, con una media de 146,3 cabezas por rebaño, siendo en el ovino las explotaciones de menos de 500 cabezas el 96,5\%

17. NAVALPOTRO JIMÉNEZ, P. op. cit. p. 130. 
del total, agrupando el $84,4 \%$ de las cabezas ${ }^{18}$. En el caso del caprino la explotación media tenía 15,8 cabezas, concentrando las explotaciones de menos de $25 \mathrm{C}$ cabezas el $84,9 \%$ de éstas. Según este estudio el $82,7 \%$ de las explotaciones caprinas tenían menos de 6 cabezas, agrupando el 10,3\% de éstas.

CUADRO IX-A

ESTRUCTURA DE LAS EXPLOTACIONES GANADERAS DE OVINO Y CAPRINO EN GRANADA EN 1993

\begin{tabular}{|c|c|c|c|c|c|c|c|}
\hline \multirow{2}{*}{$\begin{array}{l}\text { Tamaño del } \\
\text { rebaño }\end{array}$} & \multicolumn{2}{|c|}{ Explotaciones } & \multicolumn{3}{|c|}{ № cabezas } & \multicolumn{2}{|c|}{ Primas (Pts.) } \\
\hline & Total & $\%$ & Total & Media & $\%$ & Total & $\%$ \\
\hline$<=50 \ldots \ldots \ldots . .$. & 797 & 23,61 & 23.783 & 30 & 4,24 & 96.769 .688 & 3,86 \\
\hline 51 a $100 \ldots \ldots$. & 715 & 21,18 & 53.494 & 73 & 9,53 & 215.214 .836 & 8,59 \\
\hline 101 a $200 \ldots . .$. & 868 & 25,71 & 125.042 & 144 & 22,28 & 541.980 .251 & 21,62 \\
\hline 201 a $500 \ldots . .$. & 850 & 25,18 & 259.724 & 304 & 46,28 & 1.182 .106 .227 & 47,16 \\
\hline 501 a $1000 \ldots$ & 139 & 4,12 & 87.702 & 631 & 15,63 & 416.551 .934 & 16,62 \\
\hline$>1000$ & 7 & 0,21 & 11.517 & 1.645 & 2,05 & 53.804 .664 & 2,15 \\
\hline Total ….................... & 3.376 & 100,00 & 561.262 & 166 & 100,00 & 2.506 .427 .600 & 100,00 \\
\hline
\end{tabular}

Fuente: Listados de Explotaciones. Delegación Provincial de Agricultura y Pesca. Desarrollo Ganadero.

En el polo opuesto, sólo el 4,3\% de las explotaciones tiene más de 500 cabe. zas, que son los rebaños de profesionales ganaderos propiamente dichos, y en es. pecial los más grandes, los de más de 1.000 cabezas, que son un total de 7 en la provincia, con una subvención media próxima a los 8 millones de pts. por explo tación, aunque en este caso se trate de cooperativas de explotación ó sociedades destacando una radicada en Loja, que tiene más de 3.500 cabezas.

Los rebaños intermedios de 101-200 y 201-500 representan cada uno la $4^{\mathrm{a}}$ par te del total, con una media de 144 y 304 cabezas respectivamente. Considerado: en su conjunto son los predominantes, con el $51 \%$ de las explotaciones, el $48 \% \mathrm{~d}$ los cabezas, y acaparan el $69 \%$ de las cantidades por concepto de primas percibidas Desequilibrio porcentual monetario que se explica porque en estos rebaños la pre sencia de cabras es casi testimonial, y esto hace que el total de primas sea supe rior, ya que las ovejas reciben un $20 \%$ más de subvención. No obstante, la renta bilidad final de la ganadería debe tener siempre en cuenta, tanto los gastos di explotación como el valor de la came y de la leche obtenida, especialmente li primera que es la orientación predominante en la provincia de Granada, sin exclui la lechera.

18. GARCÍA RUIZ, A.L. op. cit. p. 188. 
La estructura resultante por tipos de ganado, diferenciando el ovino, que es el predominante del caprino, excluyendo los rebaños mixtos, que son la inmensa mayoría, difiere notablemente, según se trate de un tipo u otro. Veámoslo.

El censo total de ganado ovino asciende a 422.498 cabezas, de las cuales 78.123 , el $18,5 \%$ forman explotaciones especificas de ovino, el resto, que son la inmensa mayoría también incluyen cabezas de ganado caprino, de ahí que previamente hayamos analizado la cabaña en su conjunto.

La estructura de las explotaciones de ovino exclusivamente (cuadro IX-B), pone de relieve la existencia de tres clases de rebaño tipo, ya que los porcentajes asignados a los comprendidos ente 51 a 100 ; 101 a 200 ; y 201 a 500 son muy similares, $21 ; 22,6$; y 20 respectivamente. Hay cinco rebaños grandes, el $5 \%$, que acaparan el $22 \%$ de las cabezas y de las subvenciones. Los rebaños pequeños de menos de 50 cabezas suponen el $31 \%$, casi la tercera parte, siendo entre ellos los más representativos los que tiene entre 25 y 50 cabezas. Comparando con la estructura general recogida en el cuadro IX observamos que la importancia de los rebaños pequeños se incrementa, permaneciendo los grandes prácticamente igual. En definitiva, se produce un fraccionamiento mayor al excluir las cabezas de caprino, que normalmente forman rebaño conjuntamente con las de ovino, aunque en proporción pequeña. La mayoría de los rebaños de ovino incluyen algunas cabras porque sirven de guía y aglutinante de la manada; en cambio, los rebaños de caprino exclusivo son predominantes, ya que algo más de la mitad del censo lo forman rebaños integrados solamente por cabras, con una especialización fundamentalmente lechera.

\section{CUADRO IX-B \\ ESTRUCTURA DE LAS EXPLOTACIONES DE GANADO OVINO EN GRANADA EN 1993}

\begin{tabular}{|c|c|c|c|c|c|c|c|}
\hline \multirow{2}{*}{$\begin{array}{l}\text { Tamaño del } \\
\text { rebaño }\end{array}$} & \multicolumn{2}{|c|}{ Explotaciones } & \multicolumn{3}{|c|}{ № cabezas } & \multicolumn{2}{|c|}{ Primas (Pts.) } \\
\hline & Total & $\%$ & Total & Media & $\%$ & Total & $\%$ \\
\hline$<25$ & 64 & 12,80 & 1.081 & 16,89 & 1,38 & 5.308 .569 & 1,41 \\
\hline 25 a $50 \ldots \ldots \ldots$ & 92 & 18,40 & 3.375 & 36,68 & 4,32 & 16.153 .053 & 4,30 \\
\hline 51 a $100 \ldots \ldots$ & 106 & 21,20 & 7.886 & 74,40 & 10,09 & 37.769 .523 & 10,06 \\
\hline 101 a $200 \ldots .$. & 113 & 22,60 & 16.751 & 148,24 & 21,44 & 81.004 .428 & 21,58 \\
\hline 201 a $500 \ldots . .$. & 100 & 20,00 & 31.738 & 317,38 & 40,63 & 150.455 .730 & 40,08 \\
\hline$<500$ & 25 & 5,00 & 17.292 & 691,68 & 22,13 & 84.712 .530 & 22,57 \\
\hline Total ..................... & 500 & 100,00 & 78.123 & 156,25 & 100,00 & 375.403 .833 & 100,00 \\
\hline
\end{tabular}

Fuente: Listados de Explotaciones. Delegación Provincial de Agricultura y Pesca. Desarrollo Ganadero. 
El censo de cabras asciende a 138.764 de los cuales 73.491 , el $53 \%$, forman explotaciones específicas de caprino, el resto están incluidas en rebaños de ovino predominante. La estructura de estas explotaciones la recogemos en el cuadro IX-C.

\section{CUADRO IX-C \\ ESTRUCTURA DE LAS EXPLOTACIONES DE GANADO CAPRINO EN GRANADA EN 1993}

\begin{tabular}{|c|c|c|c|c|c|c|c|}
\hline \multirow{2}{*}{$\begin{array}{l}\text { Tamaño del } \\
\text { rebaño }\end{array}$} & \multicolumn{2}{|c|}{ Explotaciones } & \multicolumn{3}{|c|}{ № cabezas } & \multicolumn{2}{|c|}{ Primas (Pts.) } \\
\hline & Total & $\%$ & Total & Media & $\%$ & Total & $\%$ \\
\hline$<25 \ldots \ldots \ldots \ldots$ & 112 & 14,55 & 1.873 & 16,72 & 2,55 & 5.648 .994 & 2,59 \\
\hline 25 a $50 \ldots \ldots \ldots$ & 181 & 23,51 & 6.465 & 35,72 & 8,80 & 19.321 .264 & 8,85 \\
\hline 51 a $100 \ldots \ldots . .$. & 229 & 29,74 & 17.394 & 75,96 & 23,67 & 50.695 .029 & 23,21 \\
\hline 101 a $200 \ldots . .$. & 193 & 25,06 & 28.865 & 149,56 & 39,28 & 85.298 .471 & 39,06 \\
\hline 201 a $500 \ldots . .$. & 54 & 7,01 & 18.369 & 340,17 & 24,99 & 55.808 .647 & 25,55 \\
\hline$<500 \ldots \ldots \ldots \ldots$ & 1 & 0,13 & 525 & 525,00 & 0,71 & 1.625 .400 & 0,74 \\
\hline Total ..................... & 770 & 100,00 & 73.491 & 95,44 & 100,00 & 218.397 .805 & 100,00 \\
\hline
\end{tabular}

Fuente: Listados de Explotaciones. Delegación Provincial de Agricultura y Pesca. Desarrollo Ganadero.

El rebaño tipo predominante es el que tiene entre 51 a 100 cabezas, casi el $30 \%$ del total de rebaños, seguido del de 101 a 200. A efectos comparativos con la estructura general incluida en el cuadro IX, los de menos de 50 cabezas serían los más representativos, con el 38\% de la cabaña. Debemos resaltar también que los rebaños grandes quedan reducidos a uno con 525 cabezas localizado en el municipio de Zafarraya. A diferencia del ovino aquí el rebaño predominante es el intermedio, ya que de los grandes sólo hay uno y los pequeños, de menos de 50 , tienen una significación menor. Esta diferencia quizá se deba a que la especialización lechera exige un mayor número de cabezas para hacerlo rentable, y porque exige una dedicación mayor de tiempo y guarda, que en los rebaños de ovino no es tan imprescindible, dada su mayor adaptabilidad. Los rebaños formados por cabras exclusivamente se concentran en tres comarcas: La Vega, con 155 rebaños y 15.548 cabezas, con una media de 100 cabezas por rebaño; La Costa, con 85 rebaños y 10.056 cabezas, y una media de 118 cabezas/rebaño. La tercera comarca en importancia por su ganado ovino es la de Alhama, que tiene 75 rebaños y $9.181 \mathrm{ca-}$ bezas, resultando la media más elevada 122 cabezas/rebaño. En el polo opuesto, podemos decir que la comarca de Baza es la que tiene una menor presencia de rebaños de cabras, sumando 1.578 cabezas distribuidas en 31 rebaños, con una media de 31 cabezas, que no es la más reducida, ya que en Huéscar la media es de 27 , pero la cabaña es tres veces superior, 4.436 cabras en rebaños exclusivos. 
Los rebaños más grandes, con más de 250 cabras, son 55, concentrándose por orden de importancia de su rebaño total en las siguientes comarcas: Alhama, con 3.164 cabezas, distribuidas en 8 rebaños, Huéscar, con 3.102 cabezas, divididas en 9 rebaños y La vega, con 2.768 cabezas, distribuidas en 9 rebaños.

La estructura por comarcas pone de manifiesto la desigual distribución de ésta en función de las características peculiares de cada una de ellas. A efectos de análisis vamos a estudiar las dos más representativas: La Vega de Granada y otra de las altiplanicies del noreste provincial, como es la comarca de Huéscar (cuadros X y XI).

CUADRO $\mathrm{X}$

ESTRUCTURA DE LAS EXPLOTACIONES GANADERAS EN LA COMARCA DE HUÉSCAR EN 1993

\begin{tabular}{|c|c|c|c|c|c|c|c|}
\hline \multirow{2}{*}{$\begin{array}{l}\text { Tamaño del } \\
\text { rebaño }\end{array}$} & \multicolumn{2}{|c|}{ Explotaciones } & \multicolumn{3}{|c|}{$\mathrm{N}^{\circ}$ cabezas } & \multicolumn{2}{|c|}{ Primas (Pts.) } \\
\hline & Total & $\%$ & Total & Media & $\%$ & Total & $\%$ \\
\hline$<=50 \ldots \ldots \ldots . . . . .$. & 109 & 17,55 & 3.235 & 30 & 2,79 & 15.289 .446 & 2,75 \\
\hline $51-100 \ldots \ldots \ldots . .$. & 108 & 17,39 & 8.007 & 74 & 6,92 & 38.182 .719 & 6,86 \\
\hline $101-200 \ldots \ldots . .$. & 182 & 29,31 & 25.723 & 141 & 22,22 & 125.077 .932 & 22,48 \\
\hline $201-500 \ldots \ldots . . .$. & 187 & 30,11 & 57.179 & 306 & 49,40 & 272.903 .271 & 49,05 \\
\hline $501-1000 \ldots \ldots$ & 35 & 5,64 & 21.612 & 617 & 18,67 & 104.883 .117 & 18,85 \\
\hline$>1000$ & 0 & 0,00 & 0 & 0 & 0,00 & 0 & 0,00 \\
\hline 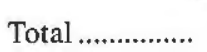 & 621 & 100,00 & 115.756 & 186 & 100,00 & 556.336 .485 & 100,00 \\
\hline
\end{tabular}

Fuente: Listados de Explotaciones. Delegación Provincial de Agricultura y Pesca. Desarrollo Ganadero.

La estructura de los rebaños en la comarca de Huéscar a finales de los setenta según García Dory ${ }^{19}$ era bastante inadecuada, ya que el $40 \%$ del número de cabezas de la comarca se encuentra en rebaños inviables. Considera que una explotación familizar viable debería tener un mínimo de 310 cabezas y $260 \mathrm{Ha}$. de tierras. La gran explotación necesitaría $627 \mathrm{Ha}$ y 750 cabezas. Según el censo aquí manejado y ampliando el número de cabezas a 350, dado el tiempo transcurrido desde que se realizó el estudio, la situación no ha variado mucho. En efecto, el 68,7\% de ellas tienen menos de 200 cabezas y considerando 350 como tamaño mínimo el porcentaje se eleva al $86,3 \%$, resultando un rebaño medio de 136 cabezas.

19. GARCÍA DORY, M.A. y MARTÍNEZ VICENTE, S. op. cit. pp. 148 


\section{CUADRO XI}

ESTRUCTURA DE LAS EXPLOTACIONES GANADERAS EN LA COMARCA DE LA VEGA EN 1993

\begin{tabular}{|c|c|c|c|c|c|c|c|}
\hline \multirow{2}{*}{$\begin{array}{l}\text { Tamaño del } \\
\text { rebaño }\end{array}$} & \multicolumn{2}{|c|}{ Explotaciones } & \multicolumn{3}{|c|}{$\mathrm{N}^{0}$ cabezas } & \multicolumn{2}{|c|}{ Primas (Pts.) } \\
\hline & Total & $\%$ & Total & Media & $\%$ & Total & $\%$ \\
\hline$<=50 \ldots \ldots \ldots . . .$. & 90 & 21,03 & 3.009 & 33 & 3,60 & 9.561 .941 & 3,02 \\
\hline $51-100 \ldots \ldots \ldots . . . . .$. & 104 & 24,30 & 7.880 & 76 & 9,44 & 23.207 .180 & 7,33 \\
\hline $101-200 \ldots . . . . . .$. & 100 & 23,36 & 14.333 & 143 & 17,17 & 47.468 .828 & 14,98 \\
\hline $201-500 \ldots \ldots \ldots$. & 107 & 25,00 & 35.150 & 328 & 42,11 & 133.474 .761 & 42,13 \\
\hline $501-1000 \ldots \ldots$ & 24 & 5,61 & 16.559 & 690 & 19,84 & 73.084 .118 & 23,07 \\
\hline$>1000 \ldots \ldots . . . . . .$. & 3 & 0,70 & 6.537 & 2.179 & 7,83 & 29.995 .434 & 9,47 \\
\hline Total ....................... & 428 & 100,00 & 83.468 & 195 & 100,00 & 316.792 .262 & 100,00 \\
\hline
\end{tabular}

Fuente: Listados de Explotaciones. Delegación Provincial de Agricultura y Pesca. Desarrollo Ganadero.

En otra comarca de las altiplanicies del noreste, como es Guadix ${ }^{20}$, la propiedad predominante es la mediana, con una media de 169 cabezas por rebaño y e] $70 \%$ de los rebaños tienen menos de 200 cabezas, localizándose la mayoría en explotaciones que tienen por centro los cortijos del secano, como Casablanca. Fuente Álamo, Olivares.

La proporción de las especies nos indica la diferente orientación técnica existente en estas dos comarcas granadinas: en la Vega el ganado ovino representa el $65 \%$ del censo; es decir, no llega a haber dos ovejas por cada cabra; en cambio, en Huéscar el $89 \%$ de los animales son ovejas; es decir hay casi 8 ovejas por cada cabra. En definitiva, esta desigual distribución del censo por especies confirma que estamos ante explotaciones con clara orientaciones diferenciadas: lechera la primera y para carne la segunda, si bien hay que hacer constatar que no existe manifiesta incompatibilidad entre ellas, aunque el total de la subvención si que es muy diferente, porque la máxima se otorga a los productores de corderos pesados, es decir a los productores de carne.

En la comarca de La Vega un hecho diferencial es el predominio del ganado caprino en los rebaños de menos de 200 cabezas, y especialmente en los más pequeños; en cambio, en la comarca de Huéscar es muy poco representativo. De ello deducimos que la orientación lechera es predominante en los pequeños rebaños de la Vega, en cambio en las altiplanicies la orientación carne es la más generalizada, casi exclusiva.

20. GÁMEZ NAVARRO, J. (1995): El espacio geográfico de Guadix: aprovechamientos agrarios, propiedad y explotación. Universidad de Granada y Fundación Caja Granada, Colección Monográfica, $n$. 211, pp. 175 
El tamaño medio del rebaño es algo inferior en las altiplanicies de Huéscar, porque predominan aquí explotaciones medianas, el $60 \%$ tienen entre 100 y 500 cabezas, no existiendo rebaños de más de 1000 cabezas; en cambio, en La Vega hay tres que superan con creces este guarismo, siendo la explotación predominante la que tiene un total comprendido entre 50-100 cabezas, seguida de cerca por las medianas. Es decir, en la comarca de La Vega la propiedad ganadera está más polarizada, y hay más explotaciones pequeñas que en Huéscar, pero también hay tres explotaciones muy grandes que tienen una media de 2.179 cabezas, entre las que destaca una Comunidad de Bienes de Loja con más de 3.500.

La estructura de las explotaciones responde a dos realidades distintas, por una parte a un medio agrícola donde el regadío es predominante, como es la comarca de La Vega, que permite el desarrollo de una actividad ganadera de reducidas dimensiones, dada la escasez de pastos; en cambio, en el noreste provincial la existencia de grandes superficie de secano y de tierras sin cultivar (monte bajo, pastos naturales) ha permitido el desarrollo de rebaños mayores para poder ser rentables. Tampoco es conveniente el rebaño excepcionalmente grande, tanto por los problemas de explotación que conlleva, como porque así se evita que la subvención disminuya, hecho que ocurre cuando el rebaño supera las 1.000 cabezas, por una norma impuesta por la U.E., de ahí que encontremos casos de rebaños grandes, pero que afectos oficiales se han distribuido entre miembros de una misma familia para evitar la disminución de la prima concedida.

En cuanto a los tipos de propiedad existentes predomina la particular o familiar, ligada o no a una explotación agrícola. Teniendo en cuenta que en los grandes rebaños es donde más predominan los tipos de propiedad distintos a la particular, en el resto, que son la mayoría, las explotaciones particulares, con el 93,83\%, son predominantes, lo que nos da una idea muy aproximada de cómo es la realidad de la propiedad. Sólo el 6,16\% restante no es propiedad privada, sino colectiva, adoptando en 4 casos la forma de C.B.; en dos la de S.A.T.; y hay además una propiedad pública (GETISA), una S.A. y otra S.L. En varios casos hemos podido detestar el fraccionamiento interesado entre padres/hijos o incluso en una C.B. del total del rebaño para que no sobrepase la cantidad máxima permitida que es de 1000 cabezas en zonas desfavorecidas. Otra constatación es la referente a la localización espacial de estas formas de propiedad distinta a la particular destacando en dos municipios de gran extensión como Loja y Huéscar.

\section{DISTRIBUCIÓN COMARCAL DE LA CABAÑA OVINA Y CAPRINA}

El censo ganadero por comarcas se distribuye como recogemos en la Figura 2 y en el cuadro XII, en el que comparamos cómo ha evolucionado el censo comarcal desde 1982. Destacan las comarcas de Huéscar y Guadix, con más de 100.000 
cabezas cada una. Las comarcas que tienen un censo más reducido son Montefríc con 16.145, y el Valle de Lecrín con 10.841. Las de Baza, Iznalloz y de la Veg tienen entre 50.000 y 100.000 cabezas; y la de Alhama, La Costa y Las Alpujarra entre 20.000 y 50.000 ; en definitiva, que el sector noreste de la provincia de Gra nada, donde predomina el paisaje vegetal caracterizado por la presencia de mont bajo, secanos y espartizales, es el que tiene una más acentuada presencia de 1 cabaña ganadera. La comarca que concentra la mayor parte del censo de ovino e Huéscar, con el $25 \%$, es decir una cuarta parte de la cabaña, seguida de Guadix Baza, con un $18 \%$ cada una.

\section{CUADRO XII}

DISTRIBUCIÓN COMARCAL DEL GANADO OVINO Y CAPRINO. AÑO 1982-1993

\begin{tabular}{|c|c|c|c|c|c|c|c|c|c|}
\hline \multirow{2}{*}{ Comarca } & \multicolumn{2}{|c|}{ Ovino 92} & \multicolumn{2}{|c|}{ Ovino 93} & \multirow{2}{*}{$\frac{93 / 82}{\%}$} & \multicolumn{2}{|c|}{ Caprino 82} & \multicolumn{2}{|c|}{ Caprino 93} \\
\hline & Total & $\%$ & Total & $\%$ & & Total & $\%$ & Total & $\%$ \\
\hline 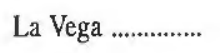 & 41.193 & 15,20 & 54.232 & 12,84 & 131,65 & 28.836 & 24,61 & 29.236 & $21,0^{\circ}$ \\
\hline 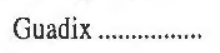 & 48.852 & 18,03 & 83.810 & 19,84 & 171,56 & 11.773 & 10,05 & 17.977 & $12,9 t$ \\
\hline 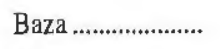 & 49.647 & 18,32 & 72.451 & 17,15 & 145,93 & 2.868 & 2,45 & 5.427 & $3,9^{\circ}$ \\
\hline Huéscar .................... & 67.201 & 24,80 & 102.621 & 24,29 & 152,71 & 8.514 & 7,27 & 13,135 & $9,4^{\circ}$ \\
\hline 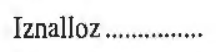 & 15.127 & 5,58 & 37.574 & 8,89 & 248,39 & 13.658 & 11,65 & 14.719 & 10,6 \\
\hline Montefrío ................. & 5.354 & 1,98 & 9.336 & 2,21 & 174,37 & 10.095 & 8,61 & 6.809 & 4,9 \\
\hline 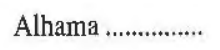 & 24.467 & 9,03 & 31.232 & 7,39 & 127,65 & 20,395 & 17,40 & 16.324 & $11,7 \mathrm{t}$ \\
\hline La Costa .................. & 1.667 & 0,62 & 7.684 & 1,82 & 460,95 & 10.098 & 8,62 & 13.322 & 9,61 \\
\hline Las Alpujarras ... & 14.949 & 5,52 & 20.636 & 4,88 & 138,04 & 7.878 & 6,72 & 13.896 & $10,0^{\circ}$ \\
\hline V. de Lecrín ........ & 2.505 & 0,92 & 2.922 & 0,69 & 116,65 & 2.992 & 2,55 & 7.919 & 5,7 \\
\hline 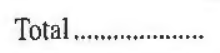 & 270.962 & 100,00 & 422.498 & 100,00 & 155,93 & 117.189 & 100,00 & 138.764 & 100,06 \\
\hline
\end{tabular}

Fuente: Los datos de 1982 se han tomado de García Ruiz, A.L.: Situación actual de la ganadería en . provincia de Granada, pp. 36. Los de 1993 de los listados utilizados como base de este artículo.

Estas tres comarcas totalizan casi el $61 \%$ del total. Por contra, hay dos comas cas que tienen una escasa participación, como son el Valle de Lecrín y la Costi con el 0,9 y $0,6 \%$ respectivamente del censo.

La evolución en esta década y salvando la dificultad que conlleva comparar datc de dos fuentes distintas, las conclusiones son: el número de animales se $\mathrm{h}$ incrementado en el $56 \%$, destacando dos comarcas por su espectacular incremer to: La Costa e Iznalloz. 


\section{FIGURA 2}

\section{COMARCAS DE GRANADA. CENSO GANADERO, 1993}

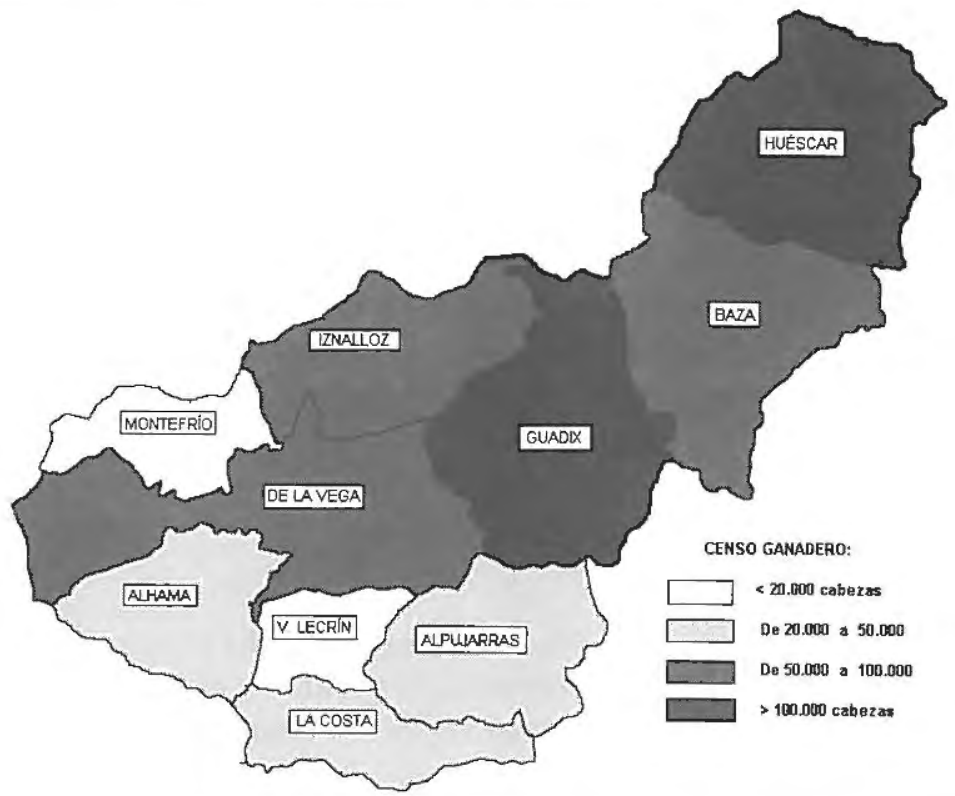

FIGURA 3

MUNICIPIOS SEGÚN SU CENSO GANADERO

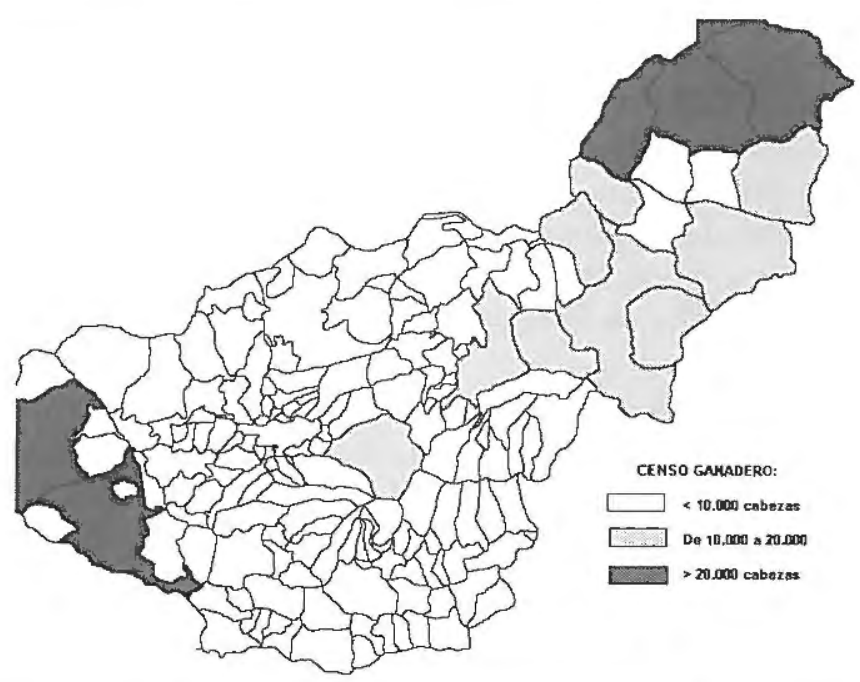


La distribución por municipios de esta importante cabaña ganadera, corrobor lo anteriormente dicho, como podemos apreciar en la Figura 3, de cuya represen tación se desprende que los municipios que tienen el censo ganadero más numero so se sitúan todos en el sector noreste de la provincia, con la excepción de Loja y Alhama, situados en el poniente, por tratarse de municipios con extensos térmi nos municipales, y de Güejar Sierra, por tratarse de un municipio de montaña. Nc podemos dejar de mencionar el hecho manifiesto de que hay un clara relación entr superficie municipal disponible para pastos y censo ganadero, lo que determin: en cierta medida que los municipios pequeños en superficie tengan también un: cabaña reducida y, que sólo en cinco casos se traduce en ausencia de cabaña cen sada, como ocurre con los casos de Cájar, Calicasas, Jun, Ogíjares y Peligros municipios situados en las proximidades de la capital.

\section{CONCLUSIONES}

- La ganadería ovina en la provincia de Granada se ha incrementado en los úl timos años debido a la puesta en marcha de las primas a este tipo de ganado, qu ha incrementado su rentabilidad de manera clara. En el último año la prima en zona desfavorecidas, en las que se incluye el $93,3 \%$ de la superficie provincial, ha as cendido a 5.360 pesetas por oveja de más de un año.

- El ganado caprino, en cambio, debido a que las primas son más bajas ha id perdiendo importancia, de hecho en la actualidad hay casi 4 cabezas de ovino po 1 de caprino, cuando a mediados de siglo estaban muy igualadas las dos cabañas

- El futuro de esta subsector ganadero va a estar muy ligado a la política d primas de la U.E., lo que no debe de repercutir en un abandono de la mejora de la razas, por la vía de la conservación y selección de aquellas mejor adaptadas a 1 orografía provincial, como son la oveja segureña, por su carne, y la cabra grañ dina, por su producción lechera.

- El sector noreste de la provincia es el que concentra la mayor parte de 1 cabaña, destacando los municipios de Huéscar, Baza y Guadix, debido a que 1 mala calidad de sus suelos ha orientado las explotaciones hacia la producció ganadera.

- La estructura ganadera aún está poco dimensionada (el 44,8\% tienen menc de 101 cabezas; y sólo el 4,3\% tienen más de 500), lo que se convierte en u handicap cara a su futuro inmediato, aunque la aparición de formas de explotació colectivas puede mejorar esta situación, pero hoy por hoy son poco representat vas. En los demás casos hay un límite máximo para recibir las ayudas totales, qu son 1000 cabezas por propietario en zonas desfavorecidas. 
- Las primas al ovino se han convertido en un gran aliciente para este tipo de ganadería, que pese a no sufrir tan intensamente la crisis ganadera que afectó a Andalucía y España en los sesenta y setenta, ha entrado en una fase expansiva, pero dependiente, en gran medida de que se mantengan tales primas.

- La comercialización de la producción de carne es el gran reto de los productores, pues es a partir de su control como se pueden obtener sustanciales ganancias. En este sentido el mercado semanal que se celebra en la ciudad de Baza cumple un papel de primer orden tanto a nivel comarcal como provincial.

\section{BIBLIOGRAFÍA MENCIONADA}

BRIZ ESCRIBANO, J. (1979): Politica de precios y estructuras en el mercado de carnes: referencia especial a las primas a la producción de añojos. Agricultura y Sociedad, $n .^{2} 11, \mathrm{pp}$. 149-206.

GÁMEZ NAVARRO, J. (1995): El espacio geográfico de Guadix: aprovechamientos agrarios, propiedad y explotación. Universidad de Granada y Fundación Caja Granada, Col. Monográfica, n. $\stackrel{\underline{0}}{211 .}$

GARCÍA DORY, M.A. (1980): La utilización de las razas autóctonas en los ecosistemas regionales como factor de ahorro energético en la ganadería española. Agricultura y Sociedad, ก. ${ }^{2} 15$, pp. 116-161.

GARCÍA DORY, M.A. y MARTÍNEZ VICENTE, S. (1988): La ganadería en España. Alianza Editorial, Madrid.

GARCÍA RUIZ, A.L. (1990): Situación de la ganadería en la provincia de Granada: composición, distribución, sistemas de explotación y comercialización de productos. Universidad de Granada.

GODOY LÓPEZ, L. (1979): La ganadería andaluza. Instituto de Desarrollo Regional. Universidad de Granada.

I.E.A. (1995): Anuario de Estadística de Andalucía. Sevilla.

I.N.E. (1994): Anuario de Estadística de España. Madrid.

INSTITUTO GALLACH (1994): Geografía de España. Vol. V. Ed. Océano, Madrid.

MAPA -SGT- (1986): Ayudas de la Comunidad Europea al sector agrario. Madrid.

NAVALPOTRO JIMÉNEZ, P. (1994): Explotaciones de ovino en los montes de Ciudad Real: viabilidad económica e impactos de la reforma de la PAC. Estudios Geográficos, n. ${ }^{\circ} 214$, pp. 109-133.

RODRÍGUEZ ZUÑIGA, M. et al. (1980): El desarrollo ganadero español: un modelo dependiente y desequilibrado. Agricultura y Sociedad, n.ํ15, pp. 165-193. 